\title{
MONITORING THE SPATIO-TEMPORAL TRAJECTORY OF URBAN AREA HOTSPOTS IN WUHAN, CHINA USING TIME-SERIES NIGHTTIME LIGHT IMAGES
}

\author{
Yuling Ruan ${ }^{1,2}$, Yanhong Zou ${ }^{1,2, *}$ \\ ${ }^{1}$ School of Geosciences and Info-Physics, Central South University, Changsha 410083, China; zouyanhong@csu.edu.cn(Y.Z.); \\ 18895327554@163.com(Y.R.) \\ ${ }^{2}$ Key Laboratory of Metallogenic Prediction of Nonferrous Metals and Geological Environment Monitoring, \\ Ministry of Education, Central South University, Changsha 410083, China
}

Commission VI, ICWG IV/III, WG VI/4

KEY WORDS: Urban area hotspot, Nighttime light imagery, DMSP/OLS, Gaussian volume model, Urbanization, Wuhan

\begin{abstract}
:
Urban area hotspots can be considered as an ideal representation of spatial heterogeneity of human activities within a city, which is susceptible to regional urban expansion pattern. However, in previous studies most researchers focused on extracting urban extent, leaving the interior variation of nighttime radiance intensity poorly explored. With the help of multi-source datasets such as DMSP/OLS (NTL), LST and NDVI, we proposed an applicable framework to identify and monitor the spatiotemporal trajectory of polycentric urban area hotspots. Firstly, the original NTL dataset were calibrated to reduce inconsistency and discontinuity. And we integrated NTL, LST as well as NDVI and established an urban index TVANUI capturing the approximate urban extents. Secondly, multi-resolution segmentation algorithm, neighborhood statistics analysis and a local-optimized threshold method were employed to get more precise urban extent with an overall accuracy above $85 \%$ and a Kappa above 0.70 . Thirdly, the urban extents were utilized as masks to get corresponding radiance intensity from calibrated NTL. Finally, we established the Gaussian volume model for each cluster and the resulting parameters were used to quantitatively depict hotspot features (i.e., intensity, morphology and centroid dynamics). All the identified urban hotspot showed our framework could successfully capture polycentric urban hotspots, whose fitting coefficients were over 0.7 . The spatiotemporal trajectory of hotspot powerfully revealed the impact of the regional urban growth pattern and planning strategies on human activities in the city of Wuhan. This study provides important insights for further studies on the relationship between the regional urbanization and human activities.
\end{abstract}

\section{INTRODUCTION}

Urbanization is a complicated phenomenon involving massive population shift, spatially expanded built-up area and adjustment of industrial structure (Cohen, 2006). Timely and accurate information on urbanization is completely of significance for land use change and environmental effect. Long term satellite observations of nighttime brightness provide an efficient way to continuously monitor human activities and socioeconomic dynamics during urbanization, especially the Defense Meteorological Satellite Program/Operational Linescan System (DMSP/OLS) nighttime light (NTL) imagery. DMSP NTL imagery therefore has been extensively used as a powerful tool for mapping urban extent despite its weaknesses of blooming and saturation (Croft, 1973; Elvidge et al., 1997; Yu et al., 2015; Xie et al., 2014; Cao et al., 2009).

Human activity, as main driven force of landscape change, has greatly influenced land-use type which results in higher intensity emerging in urban areas namely nighttime urban hotspot as an ideal proxy for describing spatial heterogeneity of human activities (Zheng et al., 2018). Particularly with deepening of urbanization, city is usually separated into several clusters from mono-centric pattern changing to polycentric one. Thus identifying the urban hotspots can not only understand the impacts of human activities, but also help decision-makers make rational development planning. However, compared to previous study, inner radiance intensity variation is unaware to be fully and deeply explored. Xiao et al. (2014) detected nighttime light imagery's centroid and analyzed the dynamic migration of centroid. Zhao et al. (2015) quantitatively analyzed the spatial differentiation and morphologic characteristics of urban core zones. Cai et al. (2017) utilized Local Moran's I to identify the main cluster within a polycentric city. Zheng et al. (2018) applied the Gaussian volume model to identify the hotspot of Hangzhou. But multi-source data has not been sufficiently integrated to detect urban extents.

Considering the land surface temperature (LST) of urban areas is generally higher than surrounding rural areas and has a strong correlation with land cover (Weng et al., 2004). While normalized differential vegetation index (NDVI) has been proven highly negatively correlated with impervious surfaces (Carlson and Arthur, 2000). In order to make better use of multi-source data to identify Wuhan's poly-centric urban structure, LST, NDVI and NTL were integrated to establish an urban index termed the Temperature and Vegetation Adjusted NTL Urban Index (TVANUI) which is closely related to urban features and mitigates the NTL blooming and saturation for mapping urban area (Zhang X et al., 2018). To further address the problem, a neighborhood statistics analysis (NSA) and local-optimized threshold method are combined to perform urban boundary extent extraction more precisely (Zou et al., 2017). And then Gaussian volume model was used to explore urban structure of Wuhan. 
Inspired and motivated by previous study, the objectives of this study were: (1) perform the urban extent extraction using multi-source datasets from 2000 to 2013; (2) identify hotspots using Gaussian volume model and depict the dynamic trajectory hotspots in Wuhan; and (3) explore the relationship between hotspot dynamics and regional urban growth.

\section{STUDY AREA AND DATA}

\subsection{Study area}

The city of Wuhan, at latitude $29^{\circ} 58^{\prime}-31^{\circ} 22^{\prime} \mathrm{N}$ and longitude $113^{\circ} 41^{\prime}-115^{\circ} 05^{\prime} \mathrm{E}$, the largest city in Central China, plays a significant role in economy, industry, culture and education. Following a polycentric pattern (He et al., 2017), Wuhan is appropriate for our study with the population and built-up urban area of 7.58 million, 10.22 million and $210 \mathrm{Km}^{2}, 534.28 \mathrm{Km}^{2}$ in 2000 and 2013 , respectively.

\subsection{Data}

The version 4 DMSP/OLS dataset obtained from the National Oceanic and Atmospheric Administration's National Geophysical Data Center (NOAA/NGDC) website (http://www.ngdc.noaa.gov/eog/dmsp.html) provided annual global composites of stable nighttime lights derived from the visible band digital numbers (DN) ranged from 0 to 63 with spatial resolution of 30 arc-seconds (approximately $1 \mathrm{~km}$ at the equator). 30-m resolution Landsat ETM+ was used to obtain land-use data downloaded from the Geospatial Data Cloud website (http://www.gscloud.cn).Noting that cloud-cover of 2009 Landsat ETM+ was unable to meet the demand even if atmospheric correction was conducted, we used the Landsat ETM+ data in 2008 to replace it. MODIS LST 1Km and NDVI $500 \mathrm{~m}$ resolution Chinese synthetic products were also downloaded from geospatial data cloud.

The point of interest (POI) data, such as shopping malls, hospitals, parks, etc., which can reflect the functional subarea certainly. A total of 167,494 POI of Wuhan in 2013 was obtained by using the web crawler technology.

\section{METHOD}

Based on time-series nighttime imagery, this study was designed to explore the distribution of radiance intensity and analyze the spatiotemporal dynamics of hotspot. The detailed methodology was illustrated as below (Figure 1).

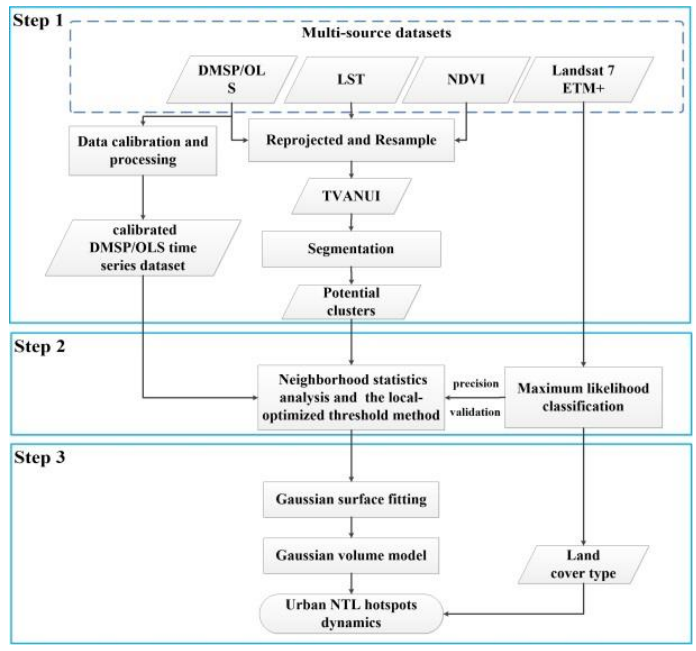

Figure 1. The flowchart of our framework

\subsection{Data Preprocessing}

Following the invariant region method proposed by Elvidge et al., (2009) and Liu et al., (2012), calibrated NTL from 2000 to 2013 were obtained. NDVI was calculated using the maximum synthesis method to highlight the spectral differences.

Radiation and atmospheric correction were applied to Landsat ETM+ imagery and the maximum likelihood method classification (MLC) was used to obtain land use type of Wuhan, the built-up derived from MLC was regarded as a reference for precision validation. LST was used to synthesize the inter-annual average image by using the average synthesis method to reduce the instability of data.

All data were projected to UTM projection in order to ensure the unity of their coordinate systems. And all data was resampled to $250-\mathrm{m}$ resolution using bi-linear interpolation to make sure there have enough numbers of pixels to meet the requirement of model fitting.

\subsection{Identifying potential clusters and Extracting the urban extent}

It was often easy to overestimate urban extent due to blooming and saturation of NTL. Many scholars have established multiple city indices to address this problem (Zhang et al., 2013 Zhang X et al., 2018). Drawing on the research results of Zhang et al. (2018), we integrated DMSP, NDVI and LST data to establish the TVANUI index which can effectively extract urban areas and enhance urban characteristics on a regional scale. The TVANUI index was expressed as Eq. (1):

$$
\text { TVANUI }=\frac{\arctan \left(\frac{\text { LST }}{\text { NDVI }}\right)}{\frac{\pi}{2}} \times \text { NTL }
$$

Data standardization was conducted to reduce inconsistency of multi-source dataset. Consequently, we obtained the 2000-2013 time-series TVANUI dataset ranged from 0 to 1 .

Multi-resolution segmentation algorithm was conducted to TVANUI. For an individual potential cluster, NSA and local-optimized threshold method were combined to further delineate more precise urban built-up area boundaries (Zou et al., 2017). The built-up urban areas in the year of 2000, 2003, 2006, 2009 and 2013 were obtained. And in the following study, nighttime urban hotspot dynamics were further analyzed in these five periods.

\subsection{Gaussian Model Fitting}

Each individual cluster following as a single-core Gaussian distribution should be treated separately. Firstly, the median filtering of $3 \times 3$ was conducted to calibrated TNL data to eliminate the influence of outliers. Secondly, the urban extent boundary was used as the mask corresponding to calibrated NTL to obtain the radiance intensity. A continuous Gaussian surface above background (blooming effect and non-urban area) can be used to describe the distribution of DN value for an individual cluster. Gaussian model was as follows Eq. (2) (Quan et al., 2014). 


$$
\begin{aligned}
F_{(x, y)}= & F_{0}+a_{0} \exp \left[-\frac{\left(\left(x-X_{0}\right) \cos \emptyset+\left(y-Y_{0}\right) \sin \emptyset\right)^{2}}{2 a_{x}{ }^{2}}\right. \\
& \left.-\frac{\left(\left(x-X_{0}\right) \sin \emptyset-\left(y-Y_{0}\right) \cos \emptyset\right)^{2}}{2 a_{y}{ }^{2}}\right]
\end{aligned}
$$

where $a_{0}$ was the magnitude, $a_{x}$ and $a_{y}$ were the half long and short axes of the Gaussian bottom ellipse, respectively. $\emptyset$ was the orientation of the Gaussian surface, and $\left(X_{0}, Y_{0}\right)$ was the center of the Gaussian bottom ellipse. $F_{0}$ was the background values defined as the minimum DN value of urban areas.

Nighttime urban hotspot can be considered as the area enclosed by the bottom ellipse. The parameters obtained from model can be used to depict hotspot. What calls for special attention was that the geometric centroid $\mathrm{O}\left(X_{0}, Y_{0}, Z_{0}\right)$ of the Gaussian volume was regarded as the hotspot centroid (Zheng et al., 2018). In fact, $\mathrm{Z}_{0}$ was irrelevant with location $\left(X_{0}, Y_{0}\right)$ and hotspot orientation $(\varnothing)$, because Gaussian volume is symmetric so that the model can be projected into a new coordinate, which could calculate $Z_{0}$ only (Zhou et al., 2011). Eq. (3) represents a simplified Gaussian surface, by using this simplified Gaussian surface, $Z_{0}$ could be solved by Eq. (4) (Quan et al., 2014). In Eq. (4), $\Omega$ represented the area of the Gaussian model. $\rho$ is a constant representing the density of Gaussian volume model.

$$
\mathrm{Z}_{(\mathrm{x}, \mathrm{y})}=\mathrm{a}_{0} \exp \left[-\frac{\mathrm{x}^{2}}{2 \mathrm{a}_{\mathrm{x}}{ }^{2}}-\frac{\mathrm{y}^{2}}{2 \mathrm{a}_{\mathrm{y}}{ }^{2}}\right]
$$

$$
Z_{0}=\frac{\iiint_{\Omega} \mathrm{z} \rho d v}{\iiint_{\Omega} \rho \mathrm{dv}}=\frac{\int_{-\mathrm{a}_{\mathrm{x}}}^{\mathrm{a}_{\mathrm{x}}}\left[\int_{-\mathrm{a}_{\mathrm{y}} \sqrt{1-\left(\mathrm{a} / \mathrm{x} / \mathrm{a}_{\mathrm{x}}\right)^{2}}}\left(\mathrm{Z}_{(\mathrm{x}, \mathrm{y})}\right)^{2} \mathrm{dy}\right] \mathrm{dx}}{2 \int_{-\mathrm{a}_{\mathrm{x}}}^{\mathrm{a}_{\mathrm{x}}}\left[\int_{-\mathrm{a}_{\mathrm{y}} \sqrt{1-\left(\mathrm{a} / \mathrm{x}_{\mathrm{y}}\right)^{2}}}^{\mathrm{a}^{1-\left(\mathrm{x} / \mathrm{a}_{\mathrm{x}}\right)^{2}}} \mathrm{Z}_{(\mathrm{x}, \mathrm{y})} \mathrm{dy}\right] \mathrm{dx}}
$$

\section{RESULTS AND DISCUSSION}

After multi-resolution segmentation, we manually merged the segments that belonged to the same potential clusters. Then NSA and local-optimized threshold method were combined to extract more precise clusters, and four clusters were identified from 2000 to 2013, there were Downtown Area (DA), Qingshan district (QS), Caidian district (CD) and Jiangxia district (JX), respectively. Actually, Xinzhou district (XZ) was firstly identified comparatively clearly in 2009. However, considering the study about dynamics of Wuhan urban hotspots was started from 2000 , the Xinzhou district was discussed in subsequent analysis. In addition, the extracted clusters were compared by that of Landsat ETM+ extraction, founding OA was above 0.85 , kappa was above 0.7 , which showed that the our extraction result was fairly reliable.

In general, model fitting results $R_{a}{ }^{2}$ of individual cluster were almost around 0.8, reference to Zheng et al., (2018), the results of the fitting $R_{a}{ }^{2}$ above 0.7 were reliable and can provide parameter support for later research.

Figure 2 showed the dynamics of the identified hotspot from 2000 to 2013. It can be easily discovered that the urban hotspots were quite consistent with the NTL data, pixels with high radiances were well enclosed by the hotspot's ellipse.
On the whole, the area presented a noticeably increasing tendency, especially for downtown area with extremely rapid urbanization and become one of the most prosperous regions (Figure 2(b)). In downtown area, the hotspot area was $199.76 \mathrm{KM}^{2}$ in 2000 and that was $522.77 \mathrm{KM}^{2}$ in 2013, although it reached $537.94 \mathrm{KM}^{2}$ in 2009 , which showed a downward trend from 2009 to 2013.Unlike built-up area in the process of urban expansion with an always rising trend, the hotspot area represented a region with a relatively higher radiance intensity at a given time, whose shape and size were easily affected by the distribution of radiance intensity and other cluster's morphology.

It can also be seen from Figure 2(b) that the hotspot of Jiangxia dist. was the smallest among all identified clusters in every year. Figure 2(c) showed that $Z_{0}$ of the downtown area was the largest among the four identified clusters, $Z_{0}$ of all four clusters showed an increasing trend from 2000 to 2013 . In the process of urbanization, the difference of $Z_{0}$ between downtown area and the other three clusters demonstrated a decreasing trend as a whole (Figure 3(a)), which indicated that the development of each cluster tended to be balanced and coordinated during the study period. It was consistent with the goal and pace of urban coordinated development driven by the urban development goal policy of Wuhan city master plan from 1996 to 2020. From Figure 3(b) that we can see, the growth rate of hotspot area was different in individual time period, generally manifesting the expansion of hotspot area. Additionally, the growth rate of downtown area was negative (-2.82\%) from 2009 to 2013, as mentioned earlier, rather than built-up area, hotspot shrank as other new hotspot appeared affecting the existing hotspot. Furthermore, except Jiangxia dist., the growth rate of the other three clusters in 2009-2013 has decreased compared with that in 2009-2006. The decline of growth rate may be related to the emergence of the 2008 rece-

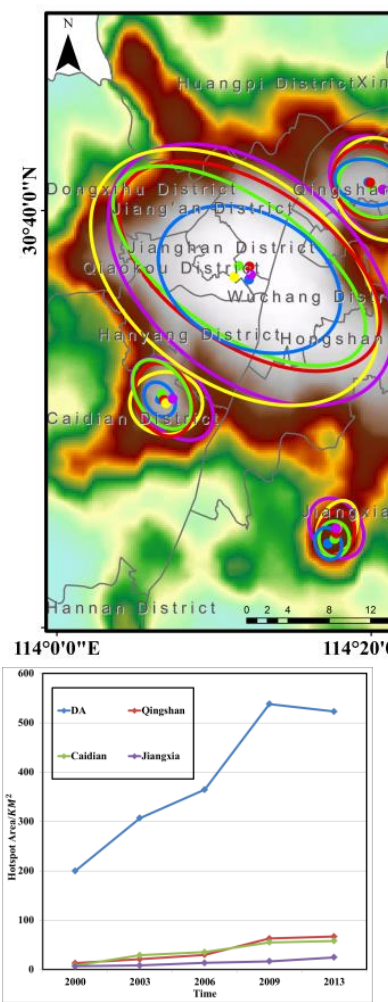

(b) (a)

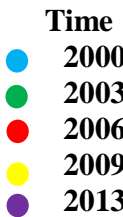

Time 2000 2003 2006 2009
Figure 2. Hotspot dynamics of study area (a); the area (b) and $Z_{0}$ (c) of each hotspot 
ssion. However, Jiangxia dist. with a superior geographical location in Wuhan metropolitan area, leading to the fact that growth rate was still rising in the period of 2009-2013 compared to that of 2006-2009.

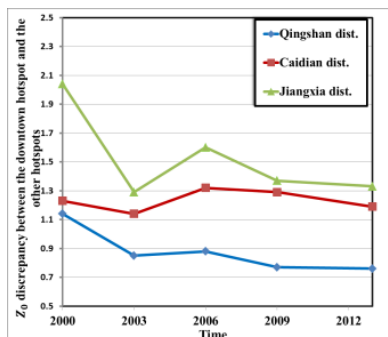

(a)

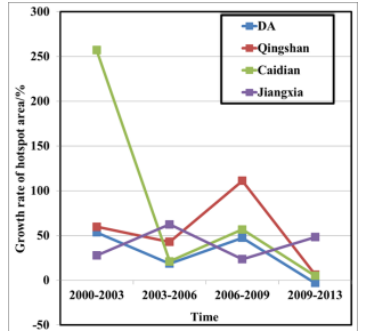

(b)
Figure $3 . Z_{0}$ discrepancy between the downtown hotspot and the other hotspots (a) and Growth rate of hotspot area (b)

The orientation of the hotspot ellipse and ratio of long and short axes reflect the discrepancy of regional urban growth. The larger the ratio was, the more serious the imbalance and inequality of region development were. Figure 4 revealed the orientation of four hotspots. The line marked with different colors represented different cluster. The standard circle was used as a reference, if the ratio of long and short axes was near to 1 , the marked color line was closer to the diameter of the circle, the more stable and coordinated the development of the cluster was.

\begin{tabular}{|l|l|l|l|l|l|}
\hline 2000 & 2003 & 2006 & 2009 & 2013 & \\
\hline & & & & & \\
\hline
\end{tabular}

Figure 4. The orientation and the ratio of $a_{x}$ and $a_{y}$ for each hotspot from 2000 to 2013

Among the four clusters, except for the Jiangxia dist., the orientation of other three clusters were more stable and did not show large fluctuations. Maybe due to the fact that Jiangxia dist. was an emerging urban area in Wuhan with many factors influencing urban development, causing more obvious fluctuation of radiance intensity. In addition, among the four clusters, the Caidian ratio of the long and short axes was relatively stable, with the lowest CV coefficient (12.04\%). On the contrary, that of Qingshan dist. was the highest (15.035\%).

The straightforward reason for hotspot dynamics was the variation of radiance intensity caused by the urban growth. As a consequence, the relationship between the area of hotspot and that of lit urban built-up as well as the relationship of $Z_{0}$ and the average radiance of lit urban built-up were discussed. Figure 5(a) showed a high correlation between the area of hotspot and that of lit urban built-up $\left(\mathrm{R}^{2}=0.9733\right)$, Figure 5 (b) also declared that average radiance had a good correlation with $Z_{0} \quad\left(\mathrm{R}^{2}=0.7192\right)$, indicating that urban hotspot was closely related to the land-use type of built-up areas, and the area of hotspot was expanding with urban growth.

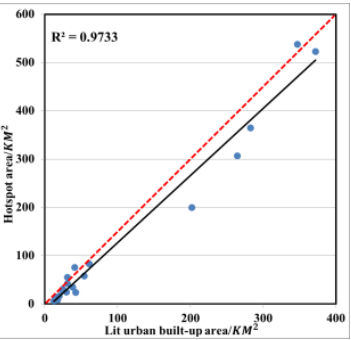

(a)

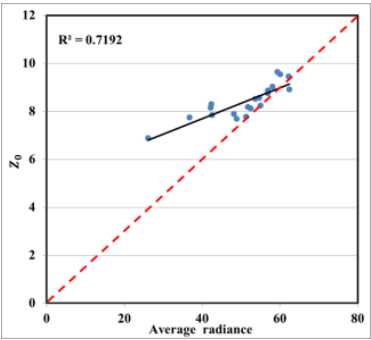

(b)
Figure 5. Linear regression between lit urban built-up area and hotspot area (a) and between average radiance and $Z_{0}$ (b) $(\mathrm{n}=$ $20)$.

With the development of Volunteered Geographic Information (VGI), POI data had also became an important data source for people to study urban spatial structure. From the perspective of land-use information and functional area, kernel density analysis was utilized to generate the POI density. The result indicated that the identified hotspots had a strong correlation with the POI density (Figure 6), the places with high POI density were included in hotspot. It was worth noting that, Xinzhou district had a relatively high POI intensity, as mentioned earlier, Xinzhou dist. was first identified clearly in 2009 from the nighttime light data, which was consistent with the results of the POI density of 2013.

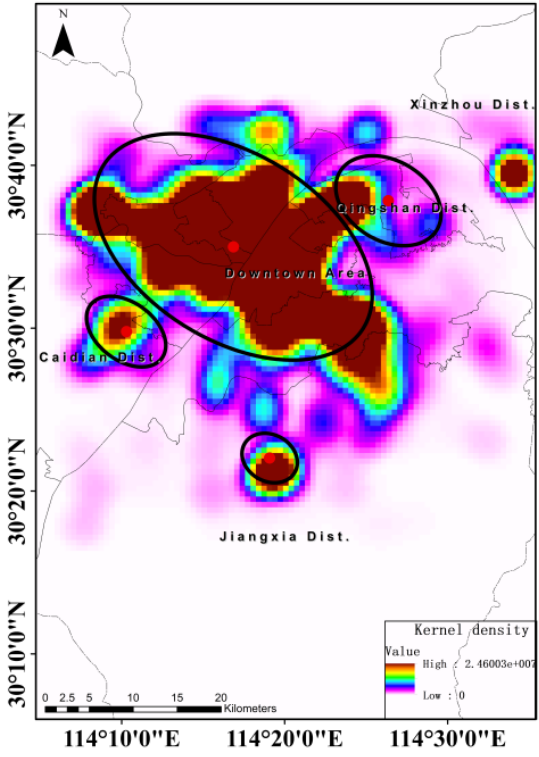

Figure 6. The kernel density of POI in 2013

Figure 7 showed the spatiotemporal trajectory of hotspot in Wuhan from 2000 to 2013, expanding as the hotspot ellipse was, the shape and orientation of downtown area as well as Qingshan district were relatively stable, as two old towns with well-established commercial center, residential area and transportation system, the urban expansion was basically along a specific direction, namely the northwest-southeast, which may be due to the influence of the Yangtze River. While Caidian and Jiangxia, as new economic development zones, the shape and orientation had a great change. Additionally, we found an obvious tendency for Caidian and Jiangxia to shift towards the downtown area. It may illustrate the fact that major hotspot might have an attractive effect on the other minor hotspots. This phenomenon occurred because of the advanc- 


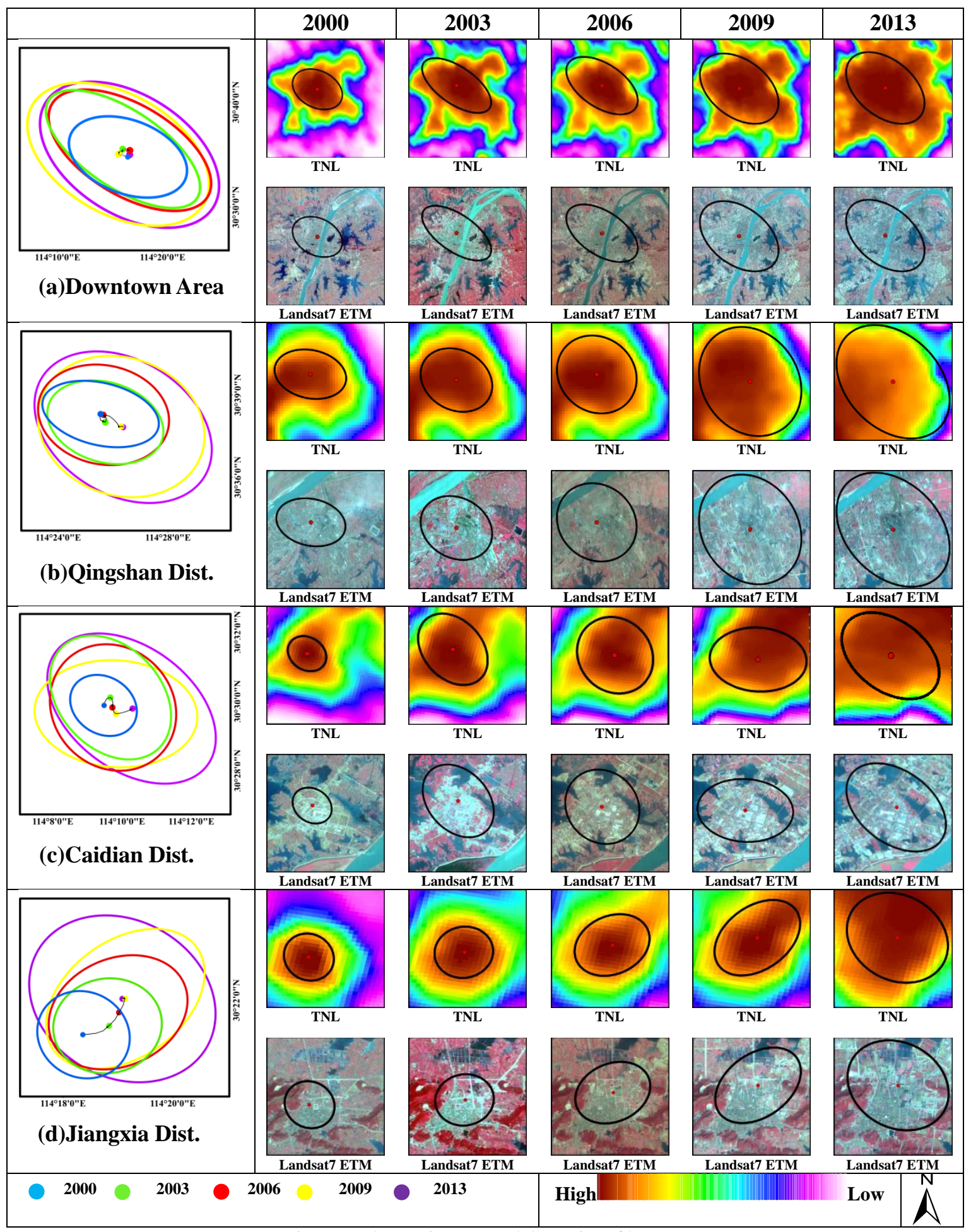

Figure 7. The spatio-temporal dynamics of hotspot

ement of traffic lines, development of Caidian district and Jiangxia district, which were adjacent to downtown area.

\section{CONCLUSION}

In order to systematically explore the nighttime radiance intensity variation so that we can get more information about changes in radiance intensity caused by urban growth, Gaussian model was utilized to identify intensified human activity areas namely urban hotspots. Spatiotemporal analysis of hotspots revealed the traits such as location, intensity, morphology, as well as migration trajectory. Meanwhile it also showed the interaction between region urban growth and human activities.

Our findings provided a robust and applicable framework to identify and monitor the spatiotemporal trajectory of polycentric urban area hotspots.

The most cities in China are experiencing the transition from mono-centric to polycentric pattern. Compared to previous, the advantages of our study are as follows. Firstly, as an effective data source for monitoring human activities, nighttime 
imageries describe the areas with intensified human activities more novelty in contrast to traditional daytime remote sensing imagery. Secondly, multi-source data were integrated to depict the urban boundary more effectively. Last but not least, the parameters obtained from Gaussian volume model can reveal the polycentric city's regional urban growth pattern more clearly than extracting the urban built-ups purely.

But there are still some limitations. Firstly, given the availability of LST data downloaded from GS Cloud since 2000, growth pattern of Wuhan before 2000 has not been studied. Besides, although NOAA released another nighttime dataset namely Suomi NPP VIIRS data in 2012, the differences in luminosity detection sensitivities, spatial resolution and over-pass time make it difficult to combine DMSP/OLS and VIIRS. In the future study, multi-source nighttime datasets should be integrated for purpose of obtaining a longer study period. Gaussian model fitting maybe be improved due to higher resolution of nighttime light data.

\section{REFERENCES}

Cai, J., Huang, B., Song, Y., 2017: Using multi-source geospatial big data to identify the structure of polycentric cities. Remote Sens. Environ.

Cao, X., Chen, J., Imura, H., Higashi, O., 2009: A SVM-based method to extract urban areas from DMSP-OLS and SPOT VGT data. Remote Sens. Environ., 113, 2205-2209.

Carlson, T.N., Arthur, S.T., 2000: The impact of land use-land cover changes due to urbanization on surface microclimate and hydrology: a satellite perspective. Global Planet. Change, 25, 49-65.

Cohen, B., 2006.Urbanization in developing countries: Current trends, future projections, and key challenges for sustainability. Technology in Society, 28, 63-80.

Croft, T., 1973. Burning waste gas in oil fields. Nature, 245, 375-376.

Elvidge, C. D., Baugh, K. E., et al., 1997: Mapping city lights with nighttime data from the DMSP Operational Linescan System. Photogrammetric Engineering \& Remote Sensing,63, 727-734.

He, J., Gao, Y., Li, C., 2017: Study on the Evolution of Multi-center Development Pattern in Wuhan. Territory \& Natural Resources Study, 6:1-7.

Liu, Z., He, C., Zhang Q., et al., 2012: Extracting the dynamics of urban expansion in China using DMSP-OLS nighttime light data from 1992 to 2008. Landscape and Urban Planning, 106(1), 62-72.

Quan, J., Chen, Y., et al., 2014: Multi-temporal trajectory of the urban heat island centroid in Beijing, China based on a Gaussian volume model. Remote Sens. Environ. 149, 33-46.

Small, C., Pozzi, F., et al., 2005: Spatial analysis of global urban extent from DMSP-OLS night lights. Remote Sens. Environ. 96, 277-91.

Weng, Q., Lu, D., Schubring, J., 2004: Estimation of land surface temperature-vegetation abundance relationship for urban heat island studies. Remote Sens. Environ. 89, 467-483.
Weng, Q., Lu, D., Schubring, J., 2004: Estimation of land surface temperature-vegetation abundance relationship for urban heat island studies. Remote Sens. Environ. 89, 467-483.

Xiao, P., Wang, X., Feng, X., et al., 2014: Detecting China's urban expansion over the past three decades using nighttime light data. IEEE J. Sel. Top. Appl. Earth Obs. Remote Sens. 7 (10), 4095-4106.

Xie, Y., Weng, Q., 2016: Updating urban extents with nighttime light imagery by using an object-based thresholding method. Remote Sensing of Environment 187, 1-13.

Yu, B., Shi, K., Hu, Y., et al., 2015: Poverty evaluation using NPPVIIRS nighttime light composite data at the county level in China. IEEE J. Sel. Top. Appl. Earth Obs. Remote Sens. 1-13.

Zhang, Q., Schaaf, C., et al., 2013: The Vegetation Adjusted NTL Urban Index: a new approach to reduce saturation and increase variation in nighttime luminosity. Remote Sens. Environ. 129, 32-41.

Zhang, X., Li, P., 2018: A temperature and vegetation adjusted NTL urban index for urban area mapping and analysis. ISPRS Journal of Photogrammetry and Remote Sensing 135, 93-111.

Zhao, N., Zhou, Y., et al., 2015: Correcting incompatible DN values and geometric errors in nighttime lights time-series images. IEEE Trans Geosci Rem Sens. 53, 2039-49.

Zhou, J., Chen, Y., et al., 2011: Maximum nighttime urban heat island (UHI) intensity simulation by integrating remotely sensed data and meteorological observations. IEEE J. Sel. Top. Appl. Earth Obs. Remote Sens. 4 (1), 138-146.

Zou, Y., Peng, H., et al., 2017: Monitoring Urban Clusters Expansion in the Middle Reaches of the Yangtze River, China, Using Time-Series Nighttime Light Images. Remote Sensing, 9 (10), 1007 